To be published in Optics Letters:

Title: $\quad$ Quasi-distributed vibration sensing using OFDR and weak reflectors

Authors: $\quad$ Daniel Leandro,Mengshi Zhu,Manuel Lopez-Amo,Hideaki Murayama

Accepted: 04 March 19

Posted $\quad 05$ March 19

Doc. ID: $\quad 355797$

(C) 2019 Optical Society of America. One print or electronic copy may be made for personal use only. Systematic reproduction and distribution, duplication of any material in this paper for a fee or for commercial purposes, or modifications of the content of this paper are prohibited." 


\title{
Quasi-distributed vibration sensing using OFDR and weak reflectors
}

\author{
Mengshi Zhu ${ }^{1,{ }^{*},+}$, Daniel Leandro ${ }^{2, *}{ }^{+}$, Manuel López-Amo ${ }^{2}$, Hideaki \\ MURAYAMA $^{3}$ \\ ${ }^{1}$ Graduate School of Engineering, The University of Tokyo, 7-3-1 Hongo, Bunkyo-ku, Tokyo, 113-8654, Japan \\ ${ }^{2}$ Dept. of Electrical, Electronic and communication Engineering, Universidad Publica de Navarra and ISC, Campus de Arrosadia, Pamplona, \\ 31006, Spain \\ ${ }^{3}$ Graduate School of Frontier Sciences, The University of Tokyo, 5-1-5 Kashiwanoha, Kashiwa-shi, Chiba, 277-8561, Japan \\ *Corresponding author: zhmsh@giso.t.u-tokyo.ac.jp, daniel.leandro@unavarra.es \\ † These authors contributed equally to this work.
}

Received XX Month XXXX; revised XX Month, XXXX; accepted XX Month XXXX; posted XX Month XXXX (Doc. ID XXXXX); published XX Month XXXX

We proposed a quasi-distributed vibration sensing technique using in-line weak reflectors and optical frequency domain reflectometry. As a result, we achieved $8 \mathrm{kHz}$ measurable vibration frequency with a $15 \sim 20 \mathrm{~cm}$ spatial resolution employing a low repetition rate $(\sim 8$ $\mathrm{Hz}$ ), Moreover, a measurable frequency of $30 \mathrm{kHz}$ was achieved for a $1.5 \mathrm{~m}$ spatial resolution. The ability of the system to determine the frequency and amplitude of several sections vibrating simultaneously is evaluated for different configurations. Because of the simple arrangement, high detectable frequency and high sensitivity, this approach is expected to be especially well suited for mechanical vibration sensing applications, particularly in medium-sized structures.

\section{(C) 2018 Optical Society of America}

http://dx.doi.org/10.1364/OL.99.099999

Fiber-optic techniques on quasi-distributed and distributed vibration/acoustic sensor (DVS/DAS) are attracting a lot of attention for their excellent potential in structural health monitoring, oil and gas pipeline security, etc [1]. Currently, the most popular approach for realizing DVS is based on the phase-sensitive optical time domain reflectometry ( $\Phi-O T D R)$ in which local phase variations are detected by using a laser source whose coherence length is longer than the spatial length of the interrogation pulse [1-2]. However, $\Phi$-OTDRs may suffer from the poor SNR from backscattering which influences their performance [3]. On the one hand, various approaches have been demonstrated to improve the signal to noise ratio (SNR) and sensitivity, such as increasing the input pulse power or implementing advanced de-noising algorithms [2, 4]. These systems can offer a high performance, at the cost of complicated setups and the use of highend/expensive equipment. On the other hand, novel DVS technologies based on optical frequency domain reflectometry (OFDR) are under development [3,5-7]. The increase of input pulse energy in OTDR based DVSs usually results in a worse spatial resolution. Therefore, the advantage of OFDR in high spatial resolution, which is only determined by the sweep range of tunable laser source (TLS), becomes very attractive. A. Am et al. demonstrated in 2014 a quasi-DVS using an array of discrete FBGs and a double OFDR interrogation scheme [3]. A year later, S. Wang et al. reported a time gated digital OFDR based DVS for demodulating the vibration induced phase changes of Rayleigh backscattering [7]. As in most approaches for vibration sensing, both systems require a very high repetition rate $\left(f_{\text {sr }}\right)$ for achieving a measurable vibration frequency up to $f_{\text {sr }} / 2$. As an alternative, Z. Ding et al. presented a long distance OFDR-based method for vibration detection by the correlation analysis of non-vibrated and vibrated Rayleigh backscattering signals [5]. In this method, the maximum detectable frequency is determined by the sweeping range of the TLS and the sample number of the correlation analysis. This makes OFDRs with low sweeping repetition rate capable of high-frequency sensing. However, this method has a lower SNR and has only achieved vibration detection at two locations. Currently, most of reported DVSs are suitable for kilometer-order sensing at meter-order spatial resolution. This performance is adequate for monitoring large structures such as oil-pipe lines, railways or bridges. However, medium-sized structures (tens of meters) such as airplane wings or helicopter blades do not require such a long range. Those DVSs have over-qualified sensing length but low spatial resolution. Therefore, a DVS system with tens of meters sensing length at a spatial resolution in centimeters order is more suitable.

In this letter, we present a new quasi-DVS technique using low repetition rate OFDR [8] and a simple weak reflector array. This technique is based on the Doppler effect in all-fiber Michelson interferometers that are sensitive to the velocity of the optical path changes instead of strain. Meanwhile, the repetition rate of the system is not limiting the maximum frequency measured. As a proof of concept, the detection of kilohertz vibrations in 30 fiber sections with a spatial resolution of $15 \sim 20 \mathrm{~cm}$ was achieved. The results show high detectable frequency ( $\mathrm{kHz}$ order), high accuracy $(<0.5 \%)$, high SNR and a linear response with respect to the magnitude of external vibration. 


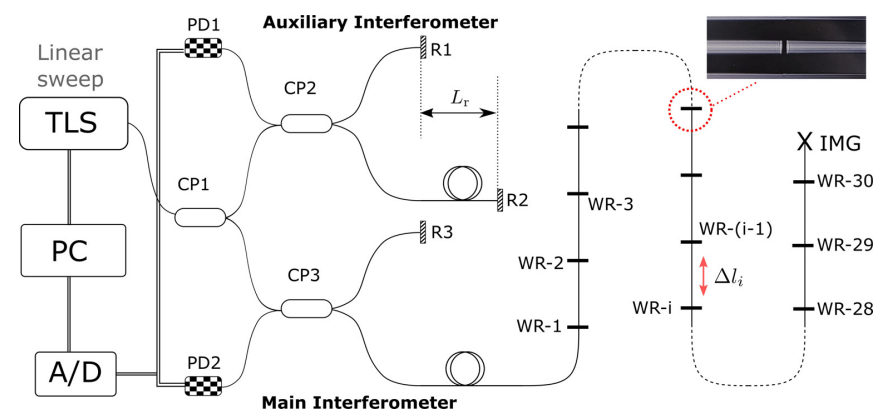

Fig. 1. Experimental setup. PD1 and PD2 are photodetectors, CP1 to CP3 are couplers, R1 to R3 are reflectors, WR-1 to WR-30 are weak reflectors, and IMG is index matching gel, respectively. $\Delta l_{i}$ is the vibration induced path change in the $i$-th section. In this work, the reflectors were formed by weak fusion splices.

Consider the OFDR system as described in Fig. 1. On the one side, an OFDR system, typically used for quasi-static strain sensing [8], essentially consists of the main interferometer, the auxiliary interferometer and the TLS. On the other, the fiber under test (FUT) is divided into sensing sections by means of weak reflectors. With a linear frequency sweep, in tens of nanometers sweeping range, the speed at wavelength domain is also approximately linear and can be calculated as

$$
\eta=\frac{\gamma \lambda_{1} \lambda_{2}}{c}
$$

where $\lambda_{1}$ and $\lambda_{2}$ are the start and end of the sweeping wavelength, $c$ is the vacuum light speed and $\gamma$ is the TLS speed in frequency domain, respectively. The clock signal generated by the auxiliary interferometer can be expressed by

$$
\begin{aligned}
& I_{D 1} \propto \cos \left(2 n_{\mathrm{eff}} L_{\mathrm{r}} k+C_{1}\right) \\
& =\cos \left(2 n_{\mathrm{eff}} L_{\mathrm{r}}\left(k_{0}+\frac{2 \pi \gamma}{c} t\right)+C_{1}\right),
\end{aligned}
$$

where $n_{\text {eff }}$ is the mode effective refractive index, $L_{\mathrm{r}}$ is the path difference between the reflectors $\mathrm{R} 1$ and $\mathrm{R} 2, k$ is the wavenumber, $k_{0}$ is the initial wavenumber, and $C_{1}$ is the randomly fluctuating optical phase which has negligible influence in meter-order range. Thus, the photo detector PD2 is triggered to be sampling at a constant wavenumber interval, $\Delta k=\pi /\left(n_{\mathrm{eff}} L_{\mathrm{r}}\right)$. Considering that the TLS performs a linear frequency sweep at the speed of $\gamma$, the corresponding time interval is $\Delta t=c /\left(2 n_{\mathrm{eff}} L_{\mathrm{r}} \gamma\right)$. In this study $L_{\mathrm{r}}=20 \mathrm{~m}$. Due to the Nyquist theorem, the maximum measurable distance is determined by this resampling process, being $10 \mathrm{~m}$. In the main interferometer, the reflected light by R3 can be expressed as

$$
E_{\mathrm{R} 3}(t)=A_{\mathrm{R} 3} \exp \left\{j\left[2 \pi f_{0} t+\pi \gamma t^{2}+C_{2}\right]\right\},
$$

where the $f_{0}$ is the initial frequency, $A_{R 3}$ is the amplitude and $C_{2}$ is the phase term of the reflected light. The reflected light by the $i$ th weak reflector can be written as

$$
E_{i}(t)=A_{i} \exp \left\{j\left[2 \pi f_{0}\left(t-\tau_{i}\right)+\pi \gamma\left(t-\tau_{i}\right)^{2}+C_{3, i}\right]\right\},
$$

where $\tau_{i}=\left(2 n_{\mathrm{eff}} Z_{i}\right) / c$ is the round trip time delay between R3 and the $i$ th weak reflector, and $C_{3, i}$ is the phase term. Assuming that $M$ vibrations occur at the FUT before WR-i, the total variation of the path, which is the function of time, can be expressed as

$$
l_{i}=\sum_{m=1}^{M} D_{m} \sin \left(2 \pi f_{\mathrm{v}, m} t+\phi_{\mathrm{v}, m}\right)+l_{i, 0},
$$

where $D_{m}$ is the amplitude, $f_{\mathrm{v}, m}$ is the vibration frequency of the $m$-th vibration $\phi_{\mathrm{v}, m}$ is the corresponding phase, and $l_{i, 0}$ is the initial path.
Therefore, the speed of the path change can be calculated as

$$
V_{i}=\frac{d l_{i}}{d t}=\sum_{m=1}^{M} 2 \pi D_{m} f_{\mathrm{v}, m} \sin \left(2 \pi f_{\mathrm{v}, m} t+\phi_{\mathrm{v}, m}\right) .
$$

Considering the Doppler effect where the optical frequency shift of the value, $n_{\text {eff }} V_{i} f_{0} / c$, will occur to the incident light, and the fact that the speed range of the TLS is limited to several nanometers per second, the corresponding reflection can be derived as

$$
\begin{aligned}
& E_{i}^{\prime}=A_{i} \exp \left\{j\left[2 \pi \int_{0}^{t-\tau_{i}}\left(f_{0}+\gamma t+\frac{n_{\mathrm{eff} V} V_{i}}{c} f_{o}\right) d t+C_{3, i}\right]\right\}, \\
& =A_{i} \exp \left\{j\left[\begin{array}{c}
2 \pi f_{0}\left(t-\tau_{i}\right)+\pi \gamma\left(t-\tau_{i}\right)^{2} \\
+\sum_{m=1}^{M} \delta_{m} \sin \left(2 \pi f_{\mathrm{v}, m} t\right)+C_{3, i}
\end{array}\right]\right\},
\end{aligned}
$$

where $\delta_{m}=n_{\mathrm{eff}} D_{m} f_{0} / c$ is the modulation index. This phenomenon can also be understood as a phase modulation. In the case of small modulation indices, by using the approximation based on the Bessel function of the first kind [9], the interference signal at PD2 can be expressed as

$$
\begin{gathered}
I_{i}=\left(E_{\mathrm{R} 3}+E_{i}\right)\left(E_{\mathrm{R} 3}+E_{i}\right)^{*} \propto \prod_{m=1}^{M} J_{0}\left(\delta_{m}\right)\left\{\cos \left[2 \pi f_{c, i} t+\phi_{c, i}\right]\right. \\
+\sum_{m=1}^{M} \frac{J_{-1}\left(\delta_{m}\right) \cos \left[2 \pi\left(f_{c, i}+f_{\mathrm{v}, m}\right) t+\phi_{-b, i}\right]}{J_{0}\left(\delta_{m}\right)} \\
\left.+\sum_{m=1}^{M} \frac{J_{1}\left(\delta_{m}\right) \cos \left[2 \pi\left(f_{c, i}-f_{\mathrm{v}, m}\right) t+\phi_{+b, i}\right]}{J_{0}\left(\delta_{m}\right)}\right\}
\end{gathered}
$$

where * denotes the complex conjugate, $J_{0, \pm 1}$ is the Bessel Function, $f_{c}=2 \gamma n_{\mathrm{eff}} z_{i} / c$ is the beat frequency of the carrier which corresponds to the location of the $i$ th weak reflector, and $\phi_{c, i}, \phi_{-b, i}, \phi_{+b, i}$ are phase terms. It is apparent that by applying the fast Fourier transform (FFT) to $I_{i}$, the centered $i$-th reflection (acting as a carrier) and vibration induced sidebands can be observed at $f_{c, i}$ and $\pm f_{\mathrm{v}, m}$, respectively. Note here that the sensing component in this approach is the variation of the optical path.

In the case of densely-spaced or continuous reflectors, such as long fiber Bragg gratings $[8,10]$ or the measurement of Rayleigh backscattering [4], the sidebands of each reflector could overlap with other carriers/sidebands due to the (almost) continuous distribution of the reflectors. That makes it difficult to demodulate the vibration information at every reflector, especially considering that the phase modulation induced by vibrations accumulates in the direction of the propagation.

To overcome this issue, instead of the fully distributed measurement along the whole sensing fiber, individual sensing sections identified by separated weak reflectors are used in this work. In this manner, each reflector and its corresponding sidebands can be clearly identified over the noise level. In the experiment, any type of reflector can be used, broad-band or wavelength-selective; such as regular FBGs, weak FBGs, fusion splices, in-line splices or fiber connectors. In our approach, weak fusion splices (inset Fig. 1) were used to interconnect the sensing sections. The length of each section is $\sim 15 \mathrm{~cm}$ showing a reflectivity $\sim 0.01 \%$. In order to generate the vibration for the validation of the system, two different transduction mechanisms have been successfully tested: PZTs and sound speakers.

As aforementioned, the phase modulation generated by vibrations forward-propagates so that every sensing section will reflect the accumulated phase change of all the previous vibrating points. In order to compensate this effect and obtain the exact vibration within each section, a demodulation process has been designed as depicted in Fig. 2. The first step for the demodulation is to perform the FFT of the beat signal so reflectors can be identified in the distance axes. Then, every reflector is windowed, obtaining a data range around each carrier peak, to be processed iteratively. Since the amplitude of the sidebands (i.e. phase modulation amplitude) is relative to the initial amplitude of the reflection, it is required the normalization of the data in the window to achieve an adequate compensation. 


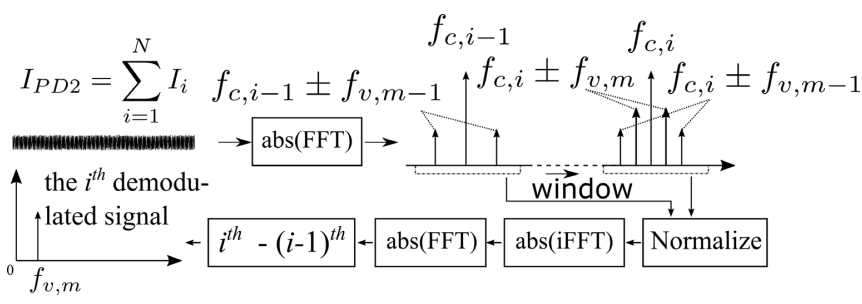

Fig. 2. Diagram of demodulation process.

In the next step, the inverse Fourier transform is performed to obtain the filtered response at each sensing fiber and then FFT is done again to the module of the obtained signal. According to the symmetric feature of FFT for real signals, the carrier frequency, $f_{c, i}$, is shifted to zero. Then, the module of the data retrieved for each section is processed to perform the compensation of the accumulated modulation by subtracting the $(i-1)$-th data trace from the $i$-th, iteratively. As a result, only the vibrations occurring at the current section remain. In the last step, the spatial separations are converted to frequencies by

$$
d f=\frac{2 \gamma n_{\mathrm{eff}}}{c} d z=\frac{2 \eta n_{\mathrm{eff}}}{\lambda_{1} \lambda_{2}} d z,
$$

where the $d f$ and $d z$ are the resolutions of frequency and distance, respectively. From the equation, it is clear that the maximum detectable frequency is determined by the length between reflectors and TLS speed as

$$
f_{\text {max }}=\frac{2 n_{\text {eff }}}{c} \gamma_{\text {max }} Z_{\text {max }}=\frac{2 n_{\text {eff }}}{\lambda_{1} \lambda_{2}} \eta_{\text {max }} Z_{\text {max }}
$$

where the subscript max represents the maximum value of corresponding variable. The OFDR trace of the FUT at position domain without vibration can be seen in Fig. 3. The TLS speed is $40 \mathrm{~nm} / \mathrm{s}$ in the wavelength range from 1550 to $1555 \mathrm{~nm}$. In other words, the repetition rate is $\sim 8 \mathrm{~Hz}$. The 30 reflectors corresponding to the sensing sections can be identified within the positions from 3.9 to $9 \mathrm{~m}$. The section length varies from 15 to $20 \mathrm{~cm}$.

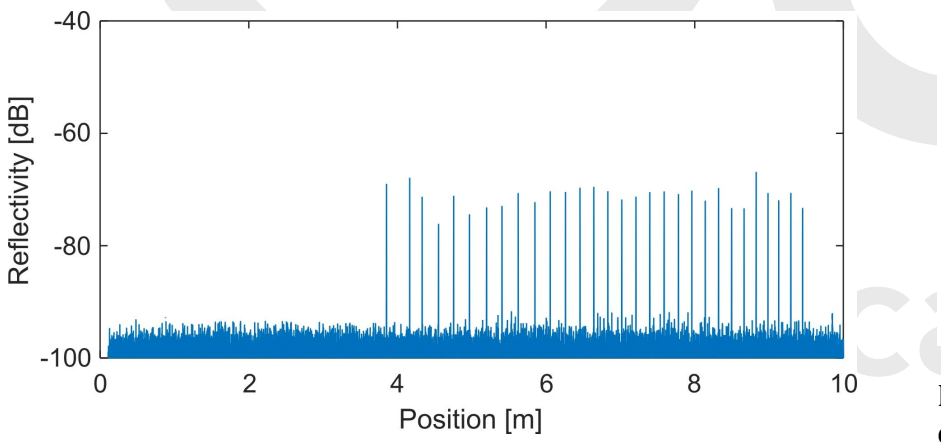

Fig. 3. OFDR trace of the thirty reflectors without vibration.

Table 1. Applied vibration conditions (SP: Speaker).

\begin{tabular}{llll|llll}
\hline Test & $\begin{array}{l}\text { Posit. } \\
{[\mathrm{m}]}\end{array}$ & $\begin{array}{l}\text { Freq. } \\
{[\mathrm{kHz}]}\end{array}$ & Type & Test & $\begin{array}{l}\text { Posit. } \\
{[\mathrm{m}]}\end{array}$ & $\begin{array}{l}\text { Freq. } \\
{[\mathrm{kHz}]}\end{array}$ & Type \\
\hline 1 & 5.8 & 1.0 & PZT & 4 & 7.0 & 0.6 & PZT \\
& & & & & 9.5 & 0.2 & SP \\
2 & 5.8 & 4.0 & PZT & 5 & 9.5 & 6.0 & PZT \\
3 & 9.5 & 0.5 & SP & 6 & 8.3 & 8.0 & SP \\
\hline
\end{tabular}

To show the demodulation performance, six different vibration conditions, given in Table 1, were applied to the FUT. In test 1, 2, 5 and 6 , the vibrations were applied by using PZT ( $\sim 0.2 \mathrm{~nm}$ amplitude). In test 3 , the vibration was applied by speaker at a sound pressure level of $80 \mathrm{dBA}$. In test 4 , the vibration at $7.0 \mathrm{~m}$ was applied by PZT $(\sim 0.2 \mathrm{~nm}$ amplitude) while at $9.5 \mathrm{~m}$ was applied by a speaker ( $80 \mathrm{dBA})$. When speaker was employed, it had no direct contact with the FUT.

In the experiment, the TLS swept from 1550 to $1555 \mathrm{~nm}$ at $40 \mathrm{~nm} / \mathrm{s}$ in tests 1 to 4 , and from 1550 to $1565 \mathrm{~nm}$ at $120 \mathrm{~nm} / \mathrm{s}$ in tests 5 and 6 . Therefore, the repetition rate was $8 \mathrm{~Hz}$. By performing the demodulation (Fig. 2), the applied distributions of vibrations have been clearly retrieved, as shown in Fig. 4 . In the figure, 15\% threshold were applied for the enhancement of contrast. It can be seen that regardless of the vibration source (PZT or speaker, with or without contact) the applied frequencies have been detected at corresponding locations.

Additionally, a measurement of chirped vibration was also performed, and the result is shown in Fig. 5. In the experiment, a frequency sweep (chirp signal) from 1.5 to $2.0 \mathrm{kHz}$ at the period of 0.05 $\mathrm{s}$ were applied to the PZT for generating a broadband vibration at 7.8 $\mathrm{m}$ (in Section 21). The TLS was set to be sweeping from 1550 to 1555 at $40 \mathrm{~nm} / \mathrm{s}$ which resulted in an $8 \mathrm{~Hz}$ repetition rate.
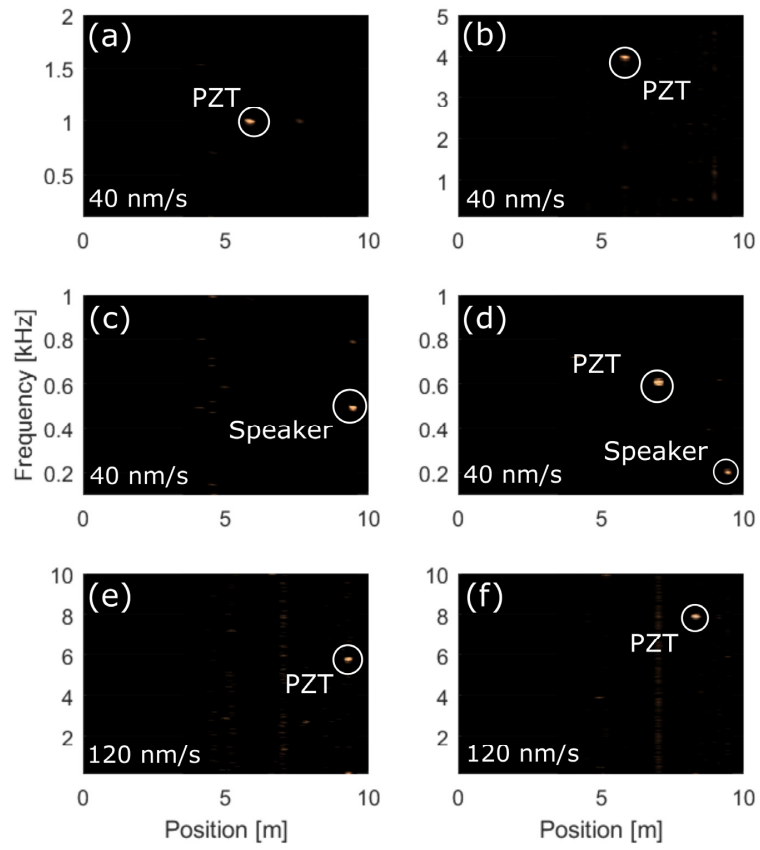

Fig. 4. Vibration information demodulated in six different cases: (a-f) correspond to tests from 1 to 6 .
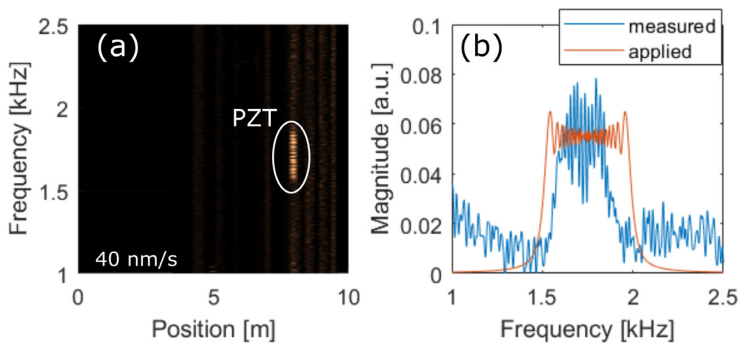

Fig. 5. Demodulated broadband vibration frequency: (a) frequency distribution, and (b) spectrum of applied chirp signal at 7.8 $\mathrm{m}$. 

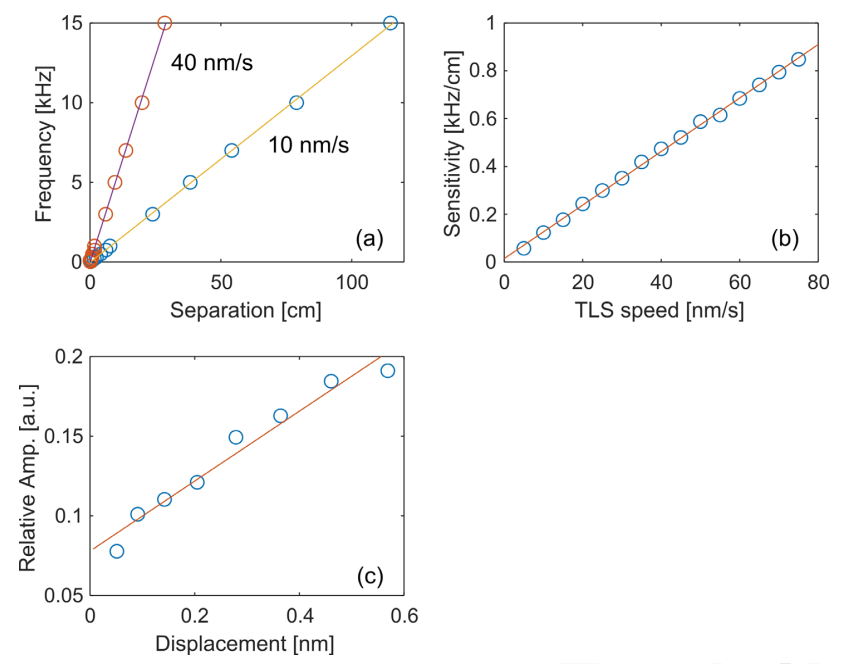

Fig. 6. Experimental calibrations: (a) frequency vs. sideband separation, (b) Sensitivity vs. TLS speed, (c) relative amplitude vs. applied displacement in nanometer.

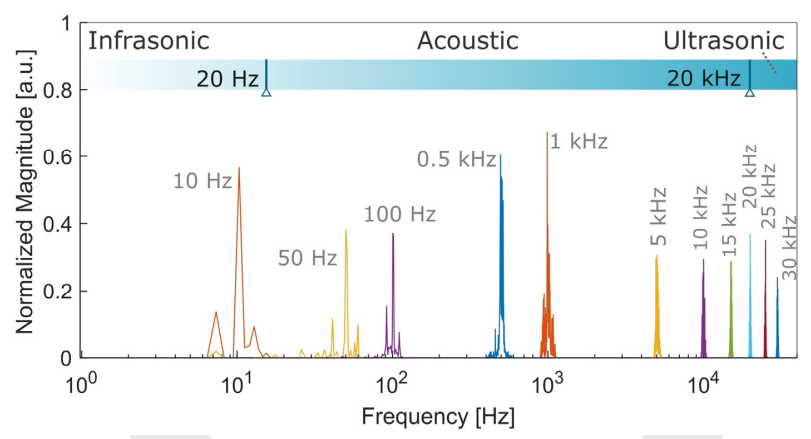

Fig. 7. Detected frequencies with $1.5 \mathrm{~m}$ section length and $40 \mathrm{~nm} / \mathrm{s}$ TLS speed. The magnitudes were normalized to give a clear view.

In addition to the demodulation, extra experiments have been performed to evaluate the ability of the system to characterize mechanical vibrations. A key property of the technique is the independence of the frequency measured with respect to the interrogation rate, which follows the theoretical relation expressed in Eq. (9) and (10). As it can be seen, the vibration frequency is defined by the distance of the sidebands to the corresponding reflection and this is also related with the tuning speed of the TLS. To verify the theory, the TLS speed was firstly set to $10 \mathrm{~nm} / \mathrm{s}$, and then the frequency response versus the sideband separation was characterized, obtaining a slope of $123 \mathrm{~Hz} / \mathrm{cm}$ (Fig. 6(a)). Meanwhile, when the TLS speed was set to be $40 \mathrm{~nm} / \mathrm{s}$, the obtained slope was $493 \mathrm{~Hz} / \mathrm{cm}$.

On the other hand, this sensitivity is directly related to the tuning speed of the TLS. Figure 6(b) shows the sensitivities in $\mathrm{kHz} / \mathrm{cm}$ at different tuning speeds (from $5 \mathrm{~nm} / \mathrm{s}$ to $75 \mathrm{~nm} / \mathrm{s}$ ). This sensitivity ranges from $60-855 \mathrm{~Hz} / \mathrm{cm}$ at TLS speeds between $5-75 \mathrm{~nm} / \mathrm{s}$. Thus, sensitivity can be tailored to match the requirements of the application by setting the TLS tuning speed.

Another important capability of the system is that the use of sidebands to perform the detection in the fiber sections allows determining the amplitude of the vibration. Since the vibration generates sidebands with an amplitude proportional to the reflection acting as carrier, this relationship remains constant even under power fluctuations. As a result, this self-referenced relationship can be used to evaluate the amplitude of the vibration. Figure 6(c) depicts the normalized amplitude measured for a single vibration event of $500 \mathrm{~Hz}$
(40 nm/s speed and $5 \mathrm{~nm}$ sweep range) for different amplitudes of applied variation of the path. In the tests, the minimum detected displacement was $0.05 \mathrm{~nm}$.

Finally, it should be said that the length of the sections only determines the maximum frequency to be measured without crosstalk. For example, at $40 \mathrm{~nm} / \mathrm{s}$ TLS speed, with $20 \mathrm{~cm}$ section length, the maximum detectable frequency is $\sim 4.8 \mathrm{kHz}$. By increasing the section length to $1.5 \mathrm{~m}$, with the same TLS speed, the maximum detectable frequency will be increased to be $\sim 36 \mathrm{kHz}$, which is demonstrated as depicted in Fig. 7. As shown, the detected frequency has already covered the whole acoustic range.

One of the main advantages of this proposal is the use of simple equipment compared to other vibration-detecting systems. It is not required to employ high-speed equipment such as modulators, fasttuning lasers, etc. Moreover, the data processing load is light enough to be performed at the measurement rate, allowing the real-time monitoring. Since the measurement rate is not related with the maximum frequency of the vibration, the performance requirements of the tunable laser source can be limited to a tuning range in the order of few nanometers with a tuning speed in the order of nm/s. On the downside, the time trace of the vibration is not recovered. However, it is retrieved the frequency and amplitude, which might be suitable for most applications.

In conclusion, we proposed a new quasi-distributed (continuous sections) fiber-optic vibration sensing technique using an in-line weak reflector array and OFDR. Different from other reported DVS/DAS which usually require very high repetition rate (directly related to the maximum measurable frequency), this approach is able to detect kilohertz frequencies at low repetition rates. Employing an OFDR initially designed for static strain sensing, driven by a demodulation algorithm for compensating the forward propagating Doppler frequency shift induced by the vibrations, it has successfully extracted the vibration information, up to $8 \mathrm{kHz}$, in 30 sensing sections at 15 to $20 \mathrm{~cm}$ spatial resolution. With larger spatial resolution $(1.5 \mathrm{~m})$, the achieved maximum detectable frequency is $30 \mathrm{kHz}$. The proposed method might have high potential in vibration sensing applications for its advantages such as simplicity, high SNR, high sensitivity, ability to obtain vibration amplitudes, high detectable frequency, etc. Due to its sensing length and spatial resolution, it is believed to be a good choice for health monitoring of structures in meter-order scale. Further work is expected to validate its performance in field testing.

Funding. This work was supported in part by the Universidad Pública de Navarra (Institute of Smart Cities) by means of a postdoctoral fellowship and project AEI/FEDERTEC2016-76021-C2-1

\section{REFERENCES}

1. Hartog, Arthur H, Chap. 9. (CRC Press, 2017).

2. Y. Lu, T. Zhu, L. Chen, and X. Bao, J. Light. Technol. Vol. 28, Issue 22, pp. 3243-3249 28, 3243-3249 (2010).

3. A. B. Am, D. Arbel, and A. Eyal, Opt. Express 22, 2299 (2014).

4. X. B. Hong, H. X. Guo, J. A. Wu, K. Xu, Y. Zuo, Y. Li, and J. Lin, Microw. Opt. Technol. Lett. 52(12), 2746-2748 (2010).

5. Z. Ding, X. S. Yao, T. Liu, Y. Du, K. Liu, Q. Han, Z. Meng, and H. Chen, Opt. Express 20, 28319 (2012).

6. L. Shiloh and A. Eyal, Opt. Express 23, 4296 (2015).

7. S. Wang, X. Fan, Q. Liu, Q., and Z. He, Op. Express 23, 33301 (2015).

8. H. Igawa, K. Ohta, T. Kasai, I. Yamaguchi, H. Murayama, and K. Kageyama, J. Solid Mech. Mater. Eng. 2, 1242-1252 (2008).

9. A. Yariv and P. Yeh, (Oxford Univ. Press, 2007).

10. H. Murayama, D. Wada, and H. Igawa, Photonic Sensors 3, 355-376 (2013). 


\section{Full-length references}

1. Hartog, Arthur H. An introduction to distributed optical fibre sensors. (CRC Press, 2017).

2. Y. Lu, T. Zhu, L. Chen, and X. Bao, "Distributed Vibration Sensor Based on Coherent Detection of Phase-OTDR," J. Light. Technol. Vol. 28, Issue 22, pp. 3243-3249 28, 3243-3249 (2010).

3. A. B. Am, D. Arbel, and A. Eyal, "OFDR with double interrogation for dynamic quasi-distributed sensing," Opt. Express 22, 2299 (2014).

4. X. B. Hong, H. X. Guo, J. A. Wu, K. Xu, Y. Zuo, Y. Li, and J. Lin, "An Intrusion Detection Sensor Based on Coherent Optical Time Domain Reflector," Microw. Opt. Technol. Lett. 52(12), 2746-2748 (2010).

5. Z. Ding, X. S. Yao, T. Liu, Y. Du, K. Liu, Q. Han, Z. Meng, and H. Chen, "Long-range vibration sensor based on correlation analysis of optical frequency-domain reflectometry signals," Opt. Express 20, 28319 (2012).

6. L. Shiloh and A. Eyal, "Distributed acoustic and vibration sensing via optical fractional Fourier transform reflectometry," Opt. Express 23, 4296 (2015).

7. Wang, S., Fan, X., Liu, Q., \& He, Z. (2015). Distributed fiber-optic vibration sensing based on phase extraction from time-gated digital OFDR. Optics Express, 23(26), 33301.

8. H. Igawa, K. Ohta, T. Kasai, I. Yamaguchi, H. Murayama, and K. Kageyama, "Distributed Measurements with a Long Gauge FBG Sensor Using Optical Frequency Domain Reflectometry," J. Solid Mech. Mater. Eng. 2, 1242-1252 (2008).

9. A. Yariv and P. Yeh, Photonics: Optical Electronics in Modern Communications (Oxford Univ. Press, 2007).

10. H. Murayama, D. Wada, and H. Igawa, "Structural health monitoring by using fiber-optic distributed strain sensors with high spatial resolution," Photonic Sensors 3, 355-376 (2013).
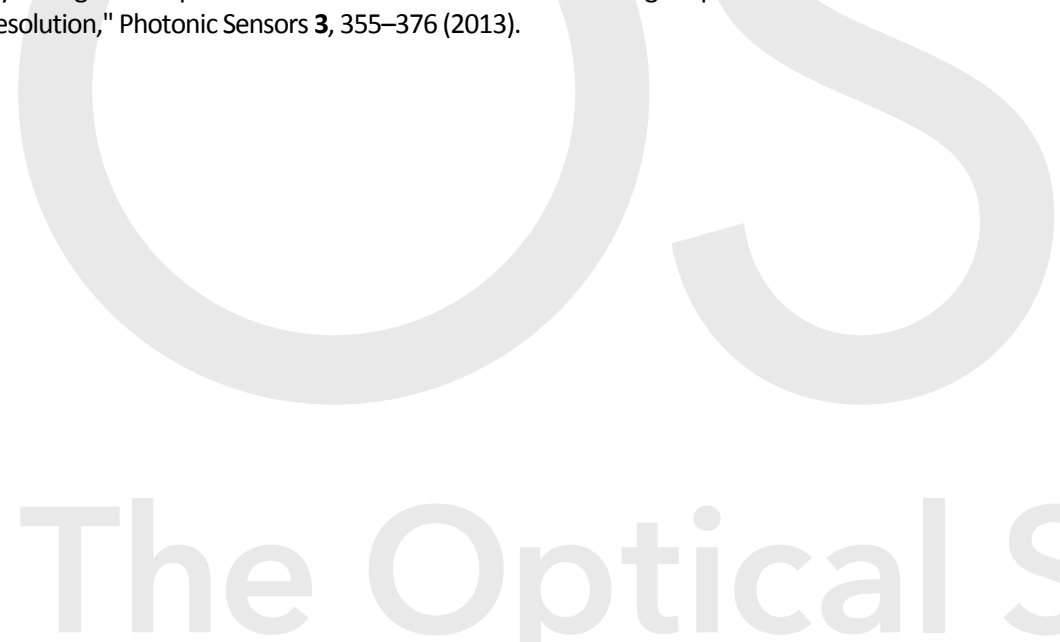